\title{
Molecular Physics of Elementary Processes Relevant to Hypersonics: Atom-Molecule, Molecule-Molecule and Atoms-Surface Processes
}

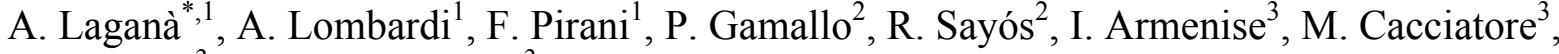 \\ F. Esposito ${ }^{3}$ and M. Rutigliano ${ }^{3}$
}

\author{
${ }^{I}$ Dipartimento di Chimica, Università di Perugia, 06100 Perugia, Italy \\ ${ }^{2}$ Departament de Química Física and Institut de Química Teórica i Computacional, Universitat de Barcelona, C. Martí i \\ Franqués 1, 08028 Barcelona, Spain \\ ${ }^{3}$ CNR-IMIP (Institute of Inorganic Methodologies and Plasmas), Via Amendola 122/D, 70126 Bari, Italy
}

\begin{abstract}
In the present chapter some prototype gas and gas-surface processes occurring within the hypersonic flow layer surrounding spacecrafts at planetary entry are discussed. The discussion is based on microscopic dynamical calculations of the detailed cross sections and rate coefficients performed using classical mechanics treatments for atoms, molecules and surfaces. Such treatment allows the evaluation of the efficiency of thermal processes (both at equilibrium and non equilibrium distributions) based on state-to-state and state specific calculations properly averaged over the population of the initial states. The dependence of the efficiency of the considered processes on the initial partitioning of energy among the various degrees of freedom is discussed.
\end{abstract}

Keywords: Classical mechanics, cross sections, dynamical calculations, rate coefficients, state-specific, state-to-state.

\section{INTRODUCTION}

The dissociated species produced in the hypersonic flows during a spacecraft planetary entry $[1,2]$ may significantly alter both the heat content of the surrounding gas layer and the heat load put on the thermal protection system (TPS) of the vehicle. This is due to both homogeneous $[3,4]$ and heterogeneous $[5,6]$ processes occurring around and at the surface of the vehicle, respectively. Such processes may be reactive (with production of new species and exchange of energy) or non reactive (with exchange of energy only). Typically, they may involve either collisions of atoms (or molecules) with molecules or collisions of atoms with surfaces.

Depending on the re-entry planet (or satellite) the involved gas phase chemical systems may vary significantly and, in the case of Earth, nitrogen (atom-diatom and diatomdiatom) systems have been, as expected, the most investigated ones [7-9]. Similar calculations have been performed also for hydrogen and oxygen systems [10-14]. As to gas surface processes, because of the fact that the characteristics of the actually used TPS materials are usually undisclosed, modeling has been performed on simpler materials like $\alpha$ - and $\beta$-quartz or $\beta$-cristobalite.

Traditionally, this kind of dynamical calculations are performed to the end of evaluating fully thermalized rate

*Address correspondence to this author at the Dipartimento di Chimica, Università di Perugia, 06100 Perugia, Italy;

Tel: +39 0755855527; Fax: +39 0755855606;

E-mail: lag@impact.dyn.unipg.it coefficients $k(T)$. More recently, however, dynamical calculation have also been performed for distinct internal (initial $i$ and final $f$ ) states to evaluate the corresponding detailed rate coefficients $k_{i, f}\left(T_{\text {col }}\right)$ (thermalized only for the collision energy $E_{\text {col }}$ distribution associated with the chosen collision temperature $T_{\text {col }}$ ). If only a subset of internal states $i^{\prime}$ (say vibrations) is explicitly considered, the remaining degrees (say rotations) can be thermalized using a separate rotational temperature $T_{\text {rot }}$ or the same as the collision one $[7,8,10,11,13]$. Such detailed computational studies bear rich information about energy disposal and state population (and as a consequence about microscopic mechanisms) especially when non equilibrium distributions are considered and rate coefficients are linked to the $\sigma_{i, f}\left(E_{c o l}\right)$ state-to-state cross section function of the $E_{\text {col }}$ collision energy.

In order to carry out accurately such detailed dynamical calculations, properly formulated atomic and molecular interactions have to be used. This makes the cost of the related massive computational campaigns feasible only by exploiting the High Performance Computing (HPC) power of the new generation of supercomputers (see PRACE [15]). However, because of the difficulty of getting timely grants able to allow a continuous and systematic performing of computational campaigns, it is expedient to exploit grid computing. Through the affiliation to the Virtual Organization (VO) COMPCHEM [16] (that gathers together several scientists operating in the field of Chemistry, Molecular and Materials Sciences and Technologies $(\mathrm{CMMST}))$ it is, in fact, possible to run massive calculations 
of the dynamical properties of the chemical systems of interest. The mentioned VO, in fact, is part of the European Grid Infrastructure (EGI) [17] and supports the use of the European grid in building collaborative computational endeavours for the study of complex molecular systems. This is grounded on the use of the various sections of the so called Grid Empowered Molecular Simulator (GEMS) [18, 19] that coordinates in a single workflow the construction of potential energy surfaces, the integration of dynamical equations and the statistical averaging of their outcomes to evaluate in a full $a b$ initio fashion rate coefficients.

Accordingly the paper is organized as follows: The basic theoretical and computational ingredients of such calculations are sketched in section 2. Three typical applications of such approach to systems relevant to the modeling of spacecraft reentry (atom-molecule, molecule-molecule and atom-surface) are discussed in sections 3, 4 and 5, respectively.

\section{THEORETICAL AND COMPUTATIONAL OUTLINES}

Kinetic models of use in gas dynamics and plasma chemistry applications usually rely on a database of thermal rate coefficients. However, in order to link them to detailed properties of both theoretical and experimental origin, $k(T)$ is better decomposed into the state-to-state rate coefficients of the intervening processes as follows

$k(T)=\sum_{i} \sum_{f} \frac{g_{i} e^{-\varepsilon_{i} / k_{B} T_{\mathrm{i} n t}}}{Q_{\mathrm{i} n t}\left(T_{\mathrm{i} n t}\right)} k_{i, f}\left(T_{\mathrm{col}}\right)$

In Eq. (1) $g_{i}$ is the multiplicity of the initial eigenstate $i$ of energy $\varepsilon_{i}, k_{B}$ is the Boltzmann constant and $Q_{\mathrm{i} n t}\left(T_{\mathrm{i} n t}\right)$ is the partition function of the internal energy at the temperature $T_{\mathrm{i} n t}$. In the case of atom-diatom processes, the roto-vibrational state $v j$ of the initial diatom to the rotovibrational state $v^{\prime} j^{\prime}$ of the final diatom detailed rate coefficient $k_{v j, v^{\prime} j^{\prime}}\left(T_{\mathrm{col}}\right)$ (i.e., $k_{i, f}\left(T_{\mathrm{col}}\right)$ term in Eq. (1)) is formulated as

$k_{v j, v^{\prime} j^{\prime}}\left(T_{\mathrm{c} o l}\right)=\sqrt{8 / k_{B}^{3} T_{\mathrm{c} o l}^{3} \pi \mu} \int_{0}^{\infty} E_{\mathrm{c} o l} \sigma_{v j, v^{\prime} j^{\prime}}\left(E_{\mathrm{c} o l}\right) e^{-E_{\mathrm{c} o l} / k_{B} T_{\mathrm{c} o l}} d E_{\mathrm{c} o l}$

In Eq. (2) $\mu$ is the reduced mass of the reactants, $E_{\mathrm{col}}$ is the collision energy of the system and $\sigma_{v j, v^{\prime} j^{\prime}}\left(E_{\mathrm{col}}\right)$ is the corresponding detailed state-to-state cross section. Detailed state-to-state cross sections are then related to the state-tostate partial (fixed value of the total angular momentum quantum number $J$ ) probabilities $P_{v j, v^{\prime} j^{\prime}}^{J}\left(E_{\mathrm{col}}\right)$ as follows

$\sigma_{v j, v^{\prime} j^{\prime}}\left(E_{\mathrm{col}}\right)=\frac{\pi}{\mathrm{k}_{v j}^{2}} \sum_{J=0}^{\infty}(2 J+1) P_{v j, v^{\prime} j^{\prime}}^{J}\left(E_{\mathrm{col}}\right)$

in which $\mathrm{k}_{v j}^{2}$ is the reactant wavenumber while $P_{v j, v^{\prime} j^{\prime}}^{J}\left(E_{\mathrm{col}}\right)$ can be derived from the exact quantum $\mathbf{S}$ matrix elements (calculated at fixed value of $J$ by integrating related exact quantum scattering equations [20] using a fully $a b$ initio potential energy surface (PES)) by means of the following relationship:
$P_{v j, v^{\prime} j^{\prime}}^{J}\left(E_{\mathrm{c} o l}\right)=\frac{1}{2 j+1} \sum_{\Lambda=-\Lambda_{\max }}^{\Lambda_{\max }} \sum_{\Lambda^{\prime}=-\Lambda_{\max }^{\prime}}^{\Lambda_{\max }^{\prime}}\left|S_{v j \Lambda, v^{\prime} j^{\prime} \Lambda^{\prime}}^{J}\left(E_{\mathrm{c} o l}\right)\right|^{2}$

with $\Lambda$ ( $\left.\Lambda^{\prime}\right)$ being the initial (final) body fixed projection of the total angular momentum $J$ and $\Lambda_{\max }=\min (j, J)$. To carry out an exact calculation of the $\mathbf{S}$ matrix elements the nuclear Hamiltonian $\hat{H}_{\mathrm{N}}$ is applied to the $\Lambda$ component of the time dependent partial wave $\Psi^{J \Lambda}(R, r, \Theta, t)$ (or similarly to the time independent analogue $\left.\Psi^{J \Lambda}(R, r, \Theta)\right)$. Most often, in order to evaluate cross sections and rate coefficients use is made of classical $(c l)$ methods, which approximate the stateto-state probability as the fraction of the trajectories starting from state $v j$ and ending in state $v^{\prime} j^{\prime}$, after all, trajectory calculations fully exploit the characteristic features of the High Throughput Computing technologies of EGI including the low level bridging of HTC with HPC [21]. This has allowed a straightforward use of the basic GEMS scheme because, as specified below in more detail, related PESs are also formulated as empirical functional forms fitted to $a b$ initio data [22]. As commented in the next sections, additional approximations are needed when moving towards more complex systems (like molecule-molecule and atom/molecule-surface systems [23]).

\section{ATOM-DIATOM SYSTEMS}

As atom-diatom case we consider here the $\mathrm{O}+\mathrm{N}_{2}(v j)$ and $\mathrm{N}+\mathrm{O}_{2}(v j)$ reactions for which the quasi-classical trajectory (QCT) method was adopted. Despite the scarcity of detailed (vibrational and rotational) experimental data to compare with, the theoretical interest for these reactions has been kept high because of their relevance in air kinetics [24]. Here we report on a systematic scan of the whole vibrational ladder in collision processes. As to the first system, the following reactive channels were considered:

$$
\begin{aligned}
& \mathrm{O}+\mathrm{N}_{2}(v j) \rightarrow \mathrm{N}+\mathrm{NO}\left(v^{\prime} j^{\prime}\right) \text { reactive channel } \\
& \mathrm{O}+\mathrm{N}_{2}(v j) \rightarrow \mathrm{O}+\mathrm{N}+\mathrm{N} \text { dissociation }
\end{aligned}
$$

though also non-reactive events (not reported here) were investigated. In the roto-vibrational energy exchange computational study, final rotation contributions are summed up, while initial rotational ones are averaged over Boltzmann distributions so as to mimic the dependence of the calculated quantities on the rotational temperature for a wide range of values. The target of such calculations were the computation of cross section values and their averaging in order to provide an estimate of the rate coefficients (inversion from rate coefficients to cross sections has shown neither to be reliable nor to be of general application [25]).

This allowed us to provide rate coefficient estimates also for non-equilibrium distributions and three body recombination rates [26], which in the case of fairly heavy species like oxygen and nitrogen are also likely to be the only contributions to recombination, because of the likely negligibility of resonant processes [26]. Concerning the O+ $\mathrm{N}_{2}$ reaction, the PESs adopted are those of Ref. [27] which are believed to be the best currently available for this system. 
The dynamics is calculated adiabatically on two PESs $\left({ }^{3} A^{\prime \prime}\right.$ ground state, ${ }^{3} A^{\prime}$ first excited state). Then the results are summed up by weighting the two contributions with an electronic factor value of $1 / 3$ [28]. For the $\mathrm{N}+\mathrm{O}_{2}$ reaction the PESs used are those of Ref. [29], with the ground state being ${ }^{2} A^{\prime}$ and the first excited one being a ${ }^{4} A^{\prime}$ state. Also in this case independent sets of results have been obtained on the two PESs which are then summed with $1 / 6$ and $1 / 3$ electronic factors respectively.

The software used for such calculations has been entirely developed by some of us [30], and then specifically adapted for distributed computations on the grid [17]. At each time step of the trajectory, a second integration is performed using a smaller step in order to check the accuracy of the integration (as discussed in Ref. [31] in this way a good balance of accuracy and computational effort is achieved, due to the possibility of efficiently adapting the timestep on the run). In order to carry out the analysis, the center of mass translational energy was binned in 1000 intervals of $10 \mathrm{meV}$ in the interval of values ranging from $1 \mathrm{meV}$ to $10 \mathrm{eV}$ so as to cover temperature intervals ranging from 300 to $10000 \mathrm{~K}$. A uniform density of 5000 trajectories per $\AA$ of impact parameter and per $\mathrm{eV}$ of translational energy has been used in the calculations leading to a total number of integrated trajectories higher than 400 millions for each chemical system. Stratified sampling has been used in all these calculations, with typical maximum impact parameters in the range 4 to $7 \AA$. The whole ladder of initial vibrational states has been scanned together with a sufficient number of rotational states in order to reliably reconstruct the whole rotational distribution by interpolation. As a consequence, one can estimate the value of the rate coefficient at all accessible rotational temperatures. The roto-vibrational ladder of levels has been calculated using the WKB approximation directly from the asymptotes of the three body potential. Related results well compare with those of more accurate calculations.

As to the accuracy and reliability of the calculations, a comparison is given in Ref. [28], where quasi-classical and wave packet results concerning the reactive channels of $\mathrm{O}+$ $\mathrm{N}_{2}$ reaction are shown together. The comparison is always excellent but at very low energies for the excited PES. A comparison of our results for the reaction channel with the well known quasi-classical results of Bose and Candler obtained on their PES [32] and with the Baulch et al. ones fitted to experimental data [33] is given in Fig. (1). From the figure it is apparent the good agreement of our results with the latter data while the agreement with the former (the theoretical ones) is not as good because they are quite lower in the whole temperature range. This, however, can be rationalized in terms of the significant differences of the two PESs and in particular by the presence in the newer surfaces of a $\mathrm{C}_{2 v}$ minimum, as discussed in Ref. [28], that lacks in the older PESs. As to dissociation, a comparison between our results and those obtained from the fit of experimental data of Shatalov [34] is given in Fig. (2). In the Figure our results appear to closely follow the latter on the whole temperature range going from 2000 to $10000 \mathrm{~K}$ confirming the reliability of the PESs used. An analogous calculation has been performed for the $\mathrm{N}+\mathrm{O}_{2}$ collision, considering both abstraction reaction and dissociation channels. Also in this case the comparison is quite good, as can be seen in the following figures. In Fig. (3) the thermal reaction rate coefficient obtained in this work for the reaction:

$$
\mathrm{N}+\mathrm{O}_{2} \rightarrow \mathrm{NO}+\mathrm{O}
$$

is shown as a red curve and compared with that of Sayós et al. obtained on the same PESs [35] (blue curve) and experimental data of Ref. [36] (green curve). The agreement looks quite satisfactory especially at intermediate temperatures. Concerning the comparison at high temperature, it is worth noting that there is a factor two error in the experimental result [36], while the theoretical result has been calculated with an approximate method [25]. As a consequence, the agreement is satisfactory. As to dissociation, literature data appears to be quite scattered. However, our results appear to be in good accord with the Shatalov results shown in Fig. (4).

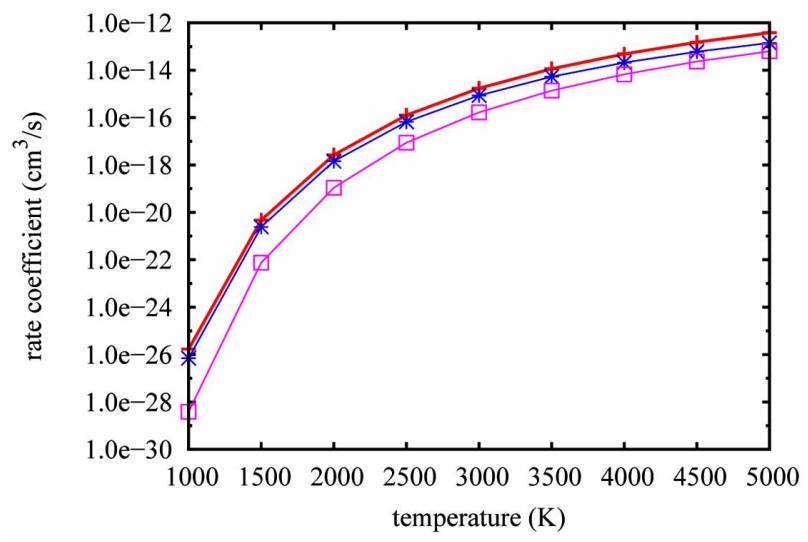

Fig. (1). Thermal rate coefficients for $\mathrm{N}_{2}+\mathrm{O} \rightarrow \mathrm{NO}+\mathrm{N}$ reaction compared with results in literature. (plus markers) this work; (asterisks) Baulch et al. [33]; (squares) Bose and Candler [32].

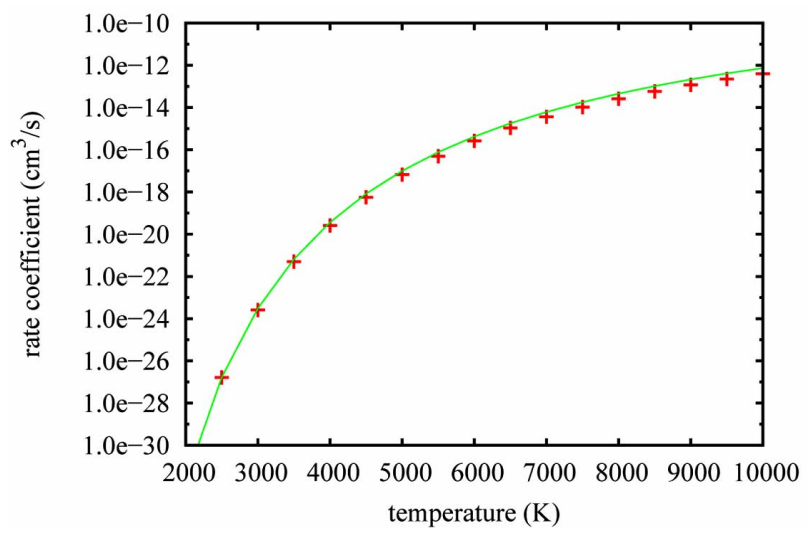

Fig. (2). Thermal rate coefficient for dissociation in $\mathrm{N}_{2}+\mathrm{O}$ collisions compared results in literature. (plus markers) this work; (solid line) Shatalov [34].

Atom diatom calculations were also performed for the $\mathrm{H}_{2}$ + He collision process that, in addition to its relevance to the study of the space vehicle entry into Jovian atmosphere [37], is one of the most important collision processes in molecular clouds [38]. The whole set of cross sections for non-reactive roto-vibrational energy exchange and dissociation, including 
quasi-bound states of reactants and products, has been calculated in the translational energy range $0.001-10 \mathrm{eV}$, with a density of 50000 trajectories per $\mathrm{eV}$ and per $\AA$ of impact parameter (with maximum value $7 \AA$ ). For this work the PES of Muchnick and Russek (MR PES) [39] has been used. Indeed, the BMP PES [40] of Boothroyd et al. is the more recently published one for the considered system and is based on a large number of ab initio points (much larger than that used for the MR PES). However, comparisons by Lee et al. [41] with experimental data have shown some problems about the fitting of the surface, while similar comparisons on the MR PES confirm its reliability.

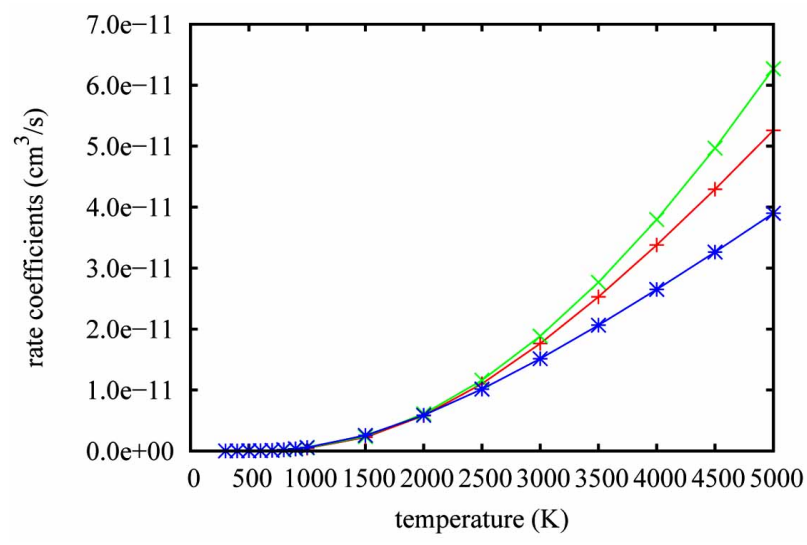

Fig. (3). Thermal rate coefficients for $\mathrm{N}+\mathrm{O}_{2} \rightarrow \mathrm{NO}+\mathrm{O}$ reaction compared with results in literature. (plus markers) this work; (cross markers) Sayós et al. [29]; (asterisks) Baulch et al. [36].

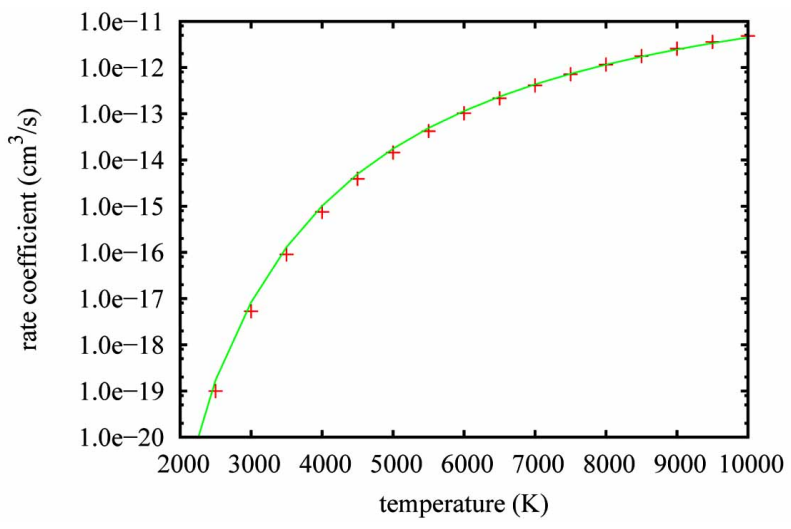

Fig. (4). Thermal rate coefficient for dissociation in $\mathrm{N}+\mathrm{O}_{2}$ collisions compared results in literature. (plus markers) this work; (solid line) Shatalov [34].

The above mentioned density of calculated trajectories is based on the integration of about 6 billions of trajectories needing approximately 9.5 years of cpu time. Again such a huge calculation has been made possible by the grid implementation of the already mentioned distributed code that runs efficiently on several serial machines even if networked on slow connections.

In order to assess the validity of the calculations, a comparison of vibrational energy exchange and dissociation are given in Figs. $(\mathbf{5}, \mathbf{6})$, respectively. The close coupling calculation of Orlikowsky [42] concerned with vibrational relaxation (from $v=1$ to $v^{\prime}=0$, including thermalized rotation) are compared in the first of them. The agreement is excellent starting from about $2000 \mathrm{~K}$. As shown in Ref. [43], in this system there is a strong correlation between rotation and vibration, and this correlation extends also to very low translational energy ranges, where the changes in rotovibrational actions can be less than one quantum, resulting in no roto-vibrational transition in the framework of quasiclassical method. This explains the failure of the method at low energy, as evidenced by Fig. (5). Concerning dissociation, on the contrary, this effect is obviously lacking. As a consequence, the agreement with approximate quantum method estimates reported in Ref. [44] is much better, as shown in Fig. (6), where the two results differ mainly in the temperature region around $10000 \mathrm{~K}$ for at most a factor two. On that ground by considering the discretization of the continuum (as well as the coupled states approximation used in Ref. [44]) and taking into account the high initial vibrational level considered, it would be not surprising to find that quasi-classical results reproduce the (not yet available) experimental data. However, it is interesting to note that, at least for this system, quasi-classical rotovibrational energy transfer results clearly fail to provide an adequate final analysis of product properties at low translational energy $(<0.1 \mathrm{eV})$, while they well reproduce quantum mechanical dissociation in the same range.

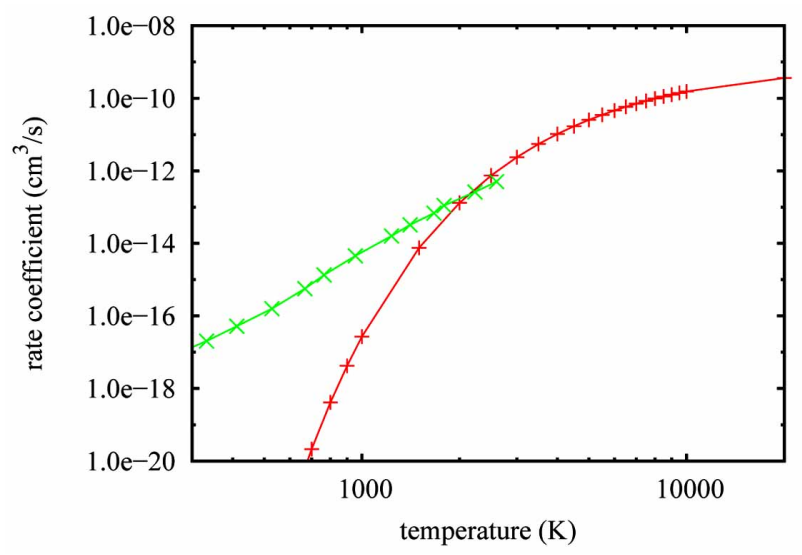

Fig. (5). Vibrational relaxation rate coefficient obtained in this work (plus markers) compared with results by Orlikowski [42] by close coupling calculations (cross markers).

\section{A MOLECULE-MOLECULE SYSTEM}

For the case of the $\mathrm{CO}_{2}-\mathrm{CO}_{2}$ collisions, which have been to some extent already illustrated in Ref. [45] and are of fundamental importance because of the widespread presence of the carbon dioxide molecule on Earth and in planetary atmospheres (such as the Martian one), the effect of vibrations and rotations of the involved molecules has to be included in the kinetic model because it strongly affects polarizabilities, charge distributions and modifies the interaction, accordingly. This is particularly true for collisions occurring at critical temperature and plasma conditions in which high internal energy levels can be significantly populated and strong excitation of the colliding molecules can be induced by an energy exchange between 
different degrees of freedom. Such effects are taken into account by using the so called bond-bond approach [46], a method for the description of the intermolecular interactions, illustrated in detail for some specific applications in Refs $[45,47-50]$. This approach apart from associating the interaction with physically meaningful properties of the collision partners, offers the advantage of allowing its flexible analytical formulation that includes explicitly the dependence of the potential functional on the internal degrees of freedom (stretching and/or bending) of the monomers and is suitable for application to even more complex cases [51,52]. To further refine the formulation of the interaction, the PES must also depend on the orientation of the molecular polarizability and on the molecular charge distribution. For the $\mathrm{CO}_{2}$ dimer case such dependence was derived in Ref. [45] where the obtained PES was also improved thanks to a comparison with ab initio data and the measured second virial coefficients. The dependence on the orientation has been treated by adopting a hyperspherical representation of the potential energy surface, by expanding the parameters of the interaction in terms of harmonic functions, a very useful practice in dealing with interaction anisotropies and for the disentangling some of the most subtle aspects of the stereodynamics of the collisions (see, e.g, $[53,54])$. It is worth to point out here that such representation indirectly takes into account also three body effects and is specifically tailored to describe two interacting $\mathrm{CO}_{2}$ molecules with high internal and rotational excitation.

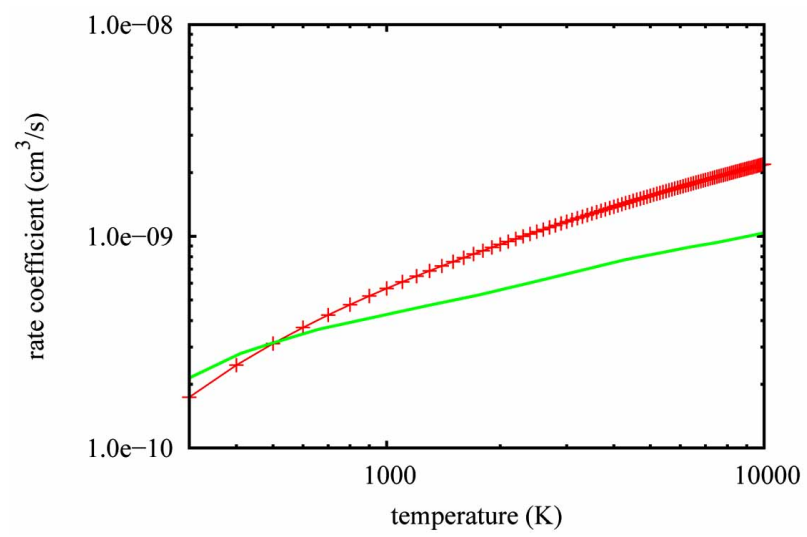

Fig. (6). Dissociation rate coefficient for $\mathrm{He}+\mathrm{H}_{2}(v=14, j=0)$ collision obtained in this work (plus markers) compared with results by Paolini et al. [44] by quantum method (solid line).

The developed intermolecular potential energy function was merged with an intramolecular potential energy function, which accounts for the energy of the two isolated molecules [55]. The resulting interaction energy function was used to carry out extended calculations of state-to-state collision cross sections using the QCT method. As already mentioned, QCT techniques are, up to date, the only viable approach to full dimensional dynamics and to the calculation of the collisional cross sections for systems having more than four atoms especially when extended data bases of state-to-state properties are needed. The combined use of the above mentioned complete PES and QCT techniques makes up a versatile computational tool for extended studies of
$\mathrm{CO}_{2}-\mathrm{CO}_{2}$ collision dynamics, which is being ported on high performance computing facilities and implemented in the European Grid Infrastructure, through the COMPCHEM VO [16], while a similar procedure is being adopted to get efficient tools to study also reactive processes (see, e.g., refs. $[56,57])$. The processes considered here are:

$$
\begin{aligned}
& \mathrm{CO}_{2}\left(v_{a 1}, v_{a 2}, v_{a 3}\right)+\mathrm{CO}_{2}\left(v_{b 1}, v_{b 2}, v_{b 3}\right) \rightarrow \\
& \mathrm{CO}_{2}\left(v_{a 1}^{\prime}, v_{a 2}^{\prime}, v_{a 3}^{\prime}\right)+\mathrm{CO}_{2}\left(v_{b 1}^{\prime}, v_{b 2}^{\prime}, v_{b 3}^{\prime}\right)
\end{aligned}
$$

where the $v_{a(b) i}(i=1,2,3)$ are the quantum labels of a normal-mode model for symmetric stretching $\left(1350 \mathrm{~cm}^{-1}\right)$, bending $\left(678 \mathrm{~cm}^{-1}\right)$ and asymmetric stretching $\left(2388 \mathrm{~cm}^{-1}\right)$ respectively, before (unprimed) and after (primed) the collision event. At equilibrium $\mathrm{CO}_{2}$ is a linear molecule, with degenerate (ground) bending states. Bending excitation generates rotation around the O--C--O molecular axis and an additional quantum number, $l$, is needed to label the discretized total molecular angular momentum projection on the quantization axis $z$ (yet the associated energy is neglected because its magnitude is often smaller than the typical statistical error of massive QCT calculations (nearly $5 \%)$ ). Indeed, the huge amount of calculations required to build a significant set of state-to-state cross sections for kinetic models by covering a sufficiently wide range of conditions, suggests to adopt an even simpler model, imposing separability between rotations and vibrations. Accordingly, the $\mathrm{CO}_{2}$ molecule is approximated as a linear rigid rotor (though dynamical effects induced by the modification of the molecular shape occurring during the collision are in any case properly taken into account by computing the true intermolecular potential). The most efficient scheme for calculations, that fully exploits the intrinsic parallelism of the QCT method, consists in setting the initial conditions of each batch of trajectories in the following way: the collision energy $E_{c o l}$ is given a fixed value; the initial rotational angular momentum of the two molecules is selected randomly by a uniform sampling of the Boltzmann distribution corresponding to the rotational temperature. The most obvious option for a rotational temperature value is to set this temperature equal to the translational one (a typical choice for this type of massive computational campaigns [45]); the initial vibrational states of the two molecules were defined by choosing two triples of integer numbers (one for each molecule) corresponding to the $v_{a(b) 1}, v_{a(b) 2}, v_{a(b) 3}$ quantum numbers. Then initial coordinates and momenta for the relative motion are set by assigning a random value to the impact parameter $b$ in the range $\left[0, b_{\max }\right]$, where $b_{\max }$, the maximum impact parameter, was taken as a truncation limit. The molecules are then randomly oriented and the initial distance between their molecular centres of mass are set large enough to make the related interaction negligible and the rotation of each of them that of a linear rigid rotor with rotations and vibrations being uncoupled. As typical of QCT methods, the final states of the two product molecules are discretized using a binning procedure. The corresponding cross sections, which can be thought of as a target area, are directly obtained by 
multiplying probabilities by a factor $\pi b_{\max }^{2}$. In the calculation presented here, we adopted $b_{\max }=20 \AA$, in order to take full account of long range force effects.

The problem of calculating state-to-state cross sections by the QCT method rises, as a side issue, the problem of separating the internal degrees of freedom of a triatomic molecule. The energy content of each vibrational degrees of freedom must be be subsequently discretized (quantized) using a binning procedure. Quantization can be achieved using a separable normal mode harmonic model because the energies corresponding to each normal mode can be directly calculated by projecting the classical phase-space vector of the system final states onto the normal mode vector basis. The rotational compontent of the energy stored in the two bending degrees of freedom can then be extracted by giving the two corresponding normal coordinates a representation in terms of polar coordinates (as stated above, this rotationa component is neglected in our calculations) [58]. Although this simple method may lead to an inaccurate labeling of the vibrational states for highly excited molecules, this is usually of negligible impact when the quantum states lie well below the strong anharmonic region of the potential. An approximate estimate of the deviation of the harmonic model from the actual behavior of the system, can be indeed made by comparing the harmonic vibrational energy levels of $\mathrm{CO}_{2}$, for a single mode of vibration, with the ones associated with the same vibrational state of the corresponding standard Morse potential (for example, the energy difference for the symmetric stretching $v_{a 1}=11$ is about $10 \%$ [45]). Moreover, such deviations have negligible impact on final thermalized results. In fact, the assignment of the final classical state to a quantum one is actually only instrumental to the setting of an integration energy grid for the evaluation of the thermal average. In other words, a difference in the labeling of the states on which the thermal average is performed changes only the location on the energy grid of the integration points without affecting the final estimate of the integral. Attention has only to be paid when dealing with the evaluation of detailed properties in which a rigorous correspondence between states and energy values need to be adopted. There are situations in which the internal dynamics of a colliding molecule is strongly anharmonic, so that the harmonic binning approximation breaks down. This is the case, for example, of high rotational temperatures, when the rotationvibration energy exchange can highly internally excite the molecules, in which a full separation of vibrational degrees of freedom makes no longer sense and a binning based on the total molecular vibrational energy would be preferable.

Examples of the calculated QCT cross sections and probabilities for $\mathrm{CO}_{2}+\mathrm{CO}_{2}$ inelastic collisions at a collision energy of $3.47 \mathrm{eV}$ and a rotational temperature of $40000 \mathrm{~K}$ are shown in Tables $\mathbf{1}$ and $\mathbf{2}$ for colliding molecules initially set either in a ground or in an excited vibrational state. Such a high collision energy and rotational excitation of molecules, although might appear as too severe conditions, are not infrequent when modeling, for example, shock waves in re-entry studies. It can be seen that, due to the quite high amount of energy available, a large number of vibrational states are populated as an effect of the collisions, and consequently there are not dominant vibrational transitions. This behaviour of the cross sections and probabilities, consisting in a slowly decreasing trend, is quite common for collision probabilities at higher energies and rotational temperatures (the opposite would be found at low energies), and it has been also encountered in previous calculations [45].

Table 1. Final States, Cross Sections and Probabilities for the Process $\mathrm{CO}_{2}(0,0,0)+\mathrm{CO}_{2}(0,0,0)$ at Collision Energy $E_{\text {col }}=3.47 \mathrm{eV}$ and Molecular Angular Momentum Randomly Selected from a Boltzmann Distribution at $40000 \mathrm{~K}$

\begin{tabular}{|c|c|c|c|}
\hline$v_{a 1}^{\prime}, v_{a 2}^{\prime}, v_{a 3}^{\prime}$ & $v_{b 1}^{\prime}, v_{b 2}^{\prime}, v_{b 3}^{\prime}$ & Prob. & Cross Section $\left(\AA^{2}\right)$ \\
\hline $\begin{array}{lll}1 & 0 & 0\end{array}$ & $\begin{array}{lll}0 & 0 & 0\end{array}$ & 0.07426 & 103.0 \\
\hline $\begin{array}{lll}1 & 1 & 0\end{array}$ & $\begin{array}{lll}1 & 0 & 0\end{array}$ & 0.06706 & 95.0 \\
\hline $\begin{array}{lll}1 & 0 & 0\end{array}$ & $\begin{array}{lll}1 & 0 & 0\end{array}$ & 0.06179 & 86.0 \\
\hline $\begin{array}{lll}1 & 0 & 0\end{array}$ & $\begin{array}{lll}0 & 1 & 0\end{array}$ & 0.04790 & 65.0 \\
\hline $\begin{array}{lll}1 & 1 & 0\end{array}$ & $\begin{array}{lll}0 & 0 & 0\end{array}$ & 0.03779 & 53.0 \\
\hline $\begin{array}{lll}0 & 1 & 0\end{array}$ & $\begin{array}{lll}0 & 0 & 0\end{array}$ & 0.02961 & 39.0 \\
\hline 120 & $\begin{array}{lll}1 & 0 & 0\end{array}$ & 0.02781 & 39.0 \\
\hline $\begin{array}{lll}1 & 1 & 0\end{array}$ & $\begin{array}{lll}0 & 1 & 0\end{array}$ & 0.02631 & 36.0 \\
\hline $\begin{array}{lll}0 & 0 & 0\end{array}$ & $\begin{array}{lll}0 & 0 & 0\end{array}$ & 0.02258 & 32.0 \\
\hline $\begin{array}{lll}1 & 0 & 0\end{array}$ & $\begin{array}{lll}0 & 2 & 0\end{array}$ & 0.02164 & 29.0 \\
\hline $\begin{array}{lll}1 & 1 & 0\end{array}$ & $\begin{array}{lll}1 & 1 & 0\end{array}$ & 0.02078 & 29.0 \\
\hline 120 & $\begin{array}{lll}0 & 0 & 0\end{array}$ & 0.01847 & 26.0 \\
\hline $\begin{array}{lll}1 & 0 & 0\end{array}$ & $\begin{array}{lll}0 & 3 & 0\end{array}$ & 0.01898 & 26.0 \\
\hline 120 & $\begin{array}{lll}1 & 1 & 0\end{array}$ & 0.01650 & 23.0 \\
\hline $\begin{array}{lll}0 & 2 & 0\end{array}$ & $\begin{array}{lll}0 & 0 & 0\end{array}$ & 0.01448 & 20.0 \\
\hline $\begin{array}{lll}1 & 0 & 0\end{array}$ & $\begin{array}{lll}0 & 4 & 0\end{array}$ & 0.01281 & 18.1 \\
\hline $\begin{array}{lll}1 & 1 & 0\end{array}$ & $\begin{array}{lll}0 & 2 & 0\end{array}$ & 0.01213 & 17.2 \\
\hline $\begin{array}{lll}0 & 1 & 0\end{array}$ & $\begin{array}{lll}0 & 1 & 0\end{array}$ & 0.01204 & 15.4 \\
\hline 120 & $\begin{array}{lll}0 & 1 & 0\end{array}$ & 0.01157 & 15.2 \\
\hline 130 & $\begin{array}{lll}1 & 0 & 0\end{array}$ & 0.01063 & 15.0 \\
\hline $\begin{array}{lll}1 & 1 & 0\end{array}$ & $\begin{array}{lll}0 & 3 & 0\end{array}$ & 0.01028 & 14.7 \\
\hline $\begin{array}{lll}0 & 3 & 0\end{array}$ & $\begin{array}{lll}0 & 0 & 0\end{array}$ & 0.01101 & 14.5 \\
\hline $\begin{array}{lll}1 & 0 & 0\end{array}$ & $\begin{array}{lll}0 & 5 & 0\end{array}$ & 0.00844 & 12.1 \\
\hline $\begin{array}{lll}0 & 3 & 0\end{array}$ & $\begin{array}{lll}0 & 1 & 0\end{array}$ & 0.00883 & 11.9 \\
\hline $\begin{array}{lll}0 & 2 & 0\end{array}$ & $\begin{array}{lll}0 & 1 & 0\end{array}$ & 0.00810 & 10.7 \\
\hline $\begin{array}{lll}0 & 4 & 0\end{array}$ & $\begin{array}{lll}0 & 0 & 0\end{array}$ & 0.00758 & 10.4 \\
\hline $\begin{array}{lll}1 & 1 & 0\end{array}$ & $\begin{array}{lll}0 & 4 & 0\end{array}$ & 0.00698 & 10.3 \\
\hline $\begin{array}{lll}1 & 3 & 0\end{array}$ & $\begin{array}{lll}0 & 0 & 0\end{array}$ & 0.00724 & 9.8 \\
\hline 140 & $\begin{array}{lll}1 & 0 & 0\end{array}$ & 0.00617 & 9.1 \\
\hline $\begin{array}{lll}1 & 3 & 0\end{array}$ & $\begin{array}{lll}1 & 1 & 0\end{array}$ & 0.00604 & 8.5 \\
\hline
\end{tabular}




\section{ATOMS RECOMBINATION ON A SURFACE}

The atom recombination on a silica surface, as already mentioned, is also a process strongly related to the vehicle surface temperature and to the flow conditions of the surrounding gas phase. In TPSs two classes of materials are used: reusable and ablative ones [59]. The first type of materials are characterized by the fact that after atmospheric entry no property or mass changes are expected and high emissivity, low thermal conductivity and low surface catalytic efficiency are related requirements. On the contrary, the second type of materials are, instead, expected to accommodate high heating loads and rates by means of mass losses and phase changes. Two coefficients (i.e., $\gamma_{i}$ and $\beta_{i}$ ) are used to suitably quantify the wall catalytic efficiency in typical computer fluid dynamics (CFD) simulations of the aerothermodynamics of these hypersonic flights [5]. The catalytic recombination coefficient $\left(\gamma_{i}\right)$ is usually obtained from the recombination probability of the $i$ th atomic species to form diatomic molecules on the surface while the chemical energy accommodation coefficient $\left(\beta_{i}\right)$ represents the ratio of energy released to the surface per atomic recombination with respect to the maximum energy transferable. The values of these coefficients fall within the range $0 \leq \gamma_{i}, \beta_{i} \leq 1$ and depend on temperature and total/partial pressures (i.e., $\gamma_{i}(\mathrm{~T}, \mathrm{P})$ ). Although some experiments have been carried out to measure such coefficients [6], some of the related publications quote the only effective catalytic efficiency values $\left(\gamma_{e f f, i}=\gamma_{i}^{\prime}=\right.$ $\gamma_{i} \cdot \beta_{i}$ ) and only a few of them quote $\beta_{i}$ coefficients that in CFD simulations are frequently assumed to be 1 (i.e., fully energy accommodation assumption). On the contrary, theoretical investigations developed at molecular dynamics level [60] have shown that only a fraction of the total exothermicity delivered in the Eley-Rideal (E-R) recombination processes of $\mathrm{O}$ and $\mathrm{N}$ on silica surfaces is transferred to the substrate as heat flux and that the main part of that energy is disposed into rotation, vibration or translation motions of the newly formed molecules. Therefore, the energy accommodation coefficient $\beta_{i}$ associated with the recombination reaction can hardly be taken as a unit. Despite the availability of results of groundbased tests, flight tests and numerical calculations play a key role in investigating the characteristics of hypersonic flights. In particular, theoretical calculations are needed to understand the catalytic effects of TPS materials. A typical rather known TPS is the reaction-cured glass (RCG) coating used in the Shuttle high-temperature reusable surface insulation (HRSI) tiles $\left(94 \%\right.$ of $\mathrm{SiO}_{2}, 4 \%$ of $\mathrm{B}_{2} \mathrm{O}_{3}$ and $2 \%$ of $\mathrm{SiB}_{4}$ ). Silicon based-materials are usually modeled as silica surfaces (such as $\alpha$ - or $\beta$-quartz and $\beta$-cristobalite). The latter (a polymorph of silica stable at high $\mathrm{T}$ similar to those of the amorphous silica used in TPSs) has been considered in previous related theoretical studies. Frequently, microkinetic models are used to simulate the heterogeneous chemical kinetics of dissociated airflow impinging on different kinds of surfaces [61-63]. Related results are in quite good agreement with experimental data and allow the calculation of $\gamma_{i}$ and $\beta_{i}$ coefficients along with their dependence on both temperature and pressure. Due to the importance of atomic adsorption, E-R reactions become usually the main step of the parametric microkinetic models. Accordingly, in the present work, a comparative study of atomic hydrogen and atomic oxygen $\mathrm{E}-\mathrm{R}$ recombination over a $\beta$-cristobalite surface is discussed. To this end an analysis of the corresponding cross sections and the $\gamma_{E-R}$ contribution to the global $\gamma$ coefficient is carried out. $\beta$-cristobalite is the most stable silica polymorph at the high temperatures involved during the space vehicle re-entry. In order to analyze the behavior of this material during the re-entry conditions in terrestrial and jovian atmospheres, we have considered a surface model of this polymorph to study the chemical processes occurring in hypersonic conditions due to the interaction of $\mathrm{H}$ or $\mathrm{O}$ atoms with the shuttle tiles since atomic and molecular oxygen or hydrogen are the main components of terrestrial and jovian atmospheres, respectively. Therefore, this contribution presents an analysis of the catalytic effect of silica with respect to the recombination of these atomic species. In particular, we have focused on the $\mathrm{H}$ and $\mathrm{O}$ recombination occurring via the socalled E-R mechanism, according to which an impinging atom from the gas-phase recombines with an atom already adsorbed on the surface:

$$
\begin{aligned}
& \mathrm{A}_{g a s}+\text { silica } \rightarrow \mathrm{A}_{a d} * \text { silica }+\Delta E_{(1)} \\
& \mathrm{A}_{g a s}+\mathrm{A}_{a d} * \text { silica } \rightarrow \mathrm{A}_{2, g a s}(v, j)+\text { silica }+\Delta E_{(2)}
\end{aligned}
$$

where, in our case, $\mathrm{A}=\mathrm{O}$ (or $\mathrm{H}$ ) and $\Delta E_{(i)}$ is the reaction exothermicity of the considered elementary process that can be transferred to the surface by the $i$ th process. Obviously, the adsorption of an atom over the surface (i.e., process (9)) is a necessary step to produce the E-R reaction (i.e., process (10)). The dynamical results reported throughout this work have been obtained using different methodologies: the semiclassical $(s c l)$ [64, 65] and classical $(c l)$ collisional methods with a Generalized Langevin Oscillator (GLO) [66]. As previously mentioned the most critical step in molecular dynamics studies is the determination of a sufficiently accurate PES able to describe correctly the process under investigation. For the case of $\mathrm{H}, \mathrm{H}_{2}$ interaction over $\beta$ cristobalite some of us have recently developed a multidimensional PES based on electronic structure DFT calculations [67] whereas for the case of the $\mathrm{O}, \mathrm{O}_{2}$ interaction over $\beta$-cristobalite two different PESs have been developed by the two groups: a first one based on DFT cluster calculations [68] and another one based on DFT periodic boundary conditions calculations [69].

As far as the PESs of the $\mathrm{O}, \mathrm{O}_{2} / \beta$-cristobalite system is concerned, the results of Ref. [68] and Ref. [69] are in substantial agreement between them. In practice, in both studies the most active site over the silica surface with respect to the $\mathrm{O}$ atom chemisorption was found to be that on top of a Si surface atom at an equilibrium distance of $1.54 \AA$ and with a chemisorption energy of nearly $5.5 \mathrm{eV}$. In the 
case of the PES for $\mathrm{H}$ adsorption on $\beta$-cristobalite, the most active site presents the same structure as the one found for $\mathrm{O}$ adsorption over $\beta$-cristobalite but with a shorter equilibrium distance of $1.50 \AA$ and with a weaker interaction energy of $2.1 \mathrm{eV}$ [67]. An independent and detailed description and discussion of the reaction probabilities obtained for the two heterogeneous processes considered here can be found in Refs. [63, 70, 71]. Here we focus on the calculation of the reactive cross sections for the two E-R recombination reactions:

Table 2. Final States, Cross Sections and Probabilities for Collisions the Process $\mathrm{CO}_{2}(1,0,0)+\mathrm{CO}_{2}(1,0,0)$ at Collision Energy $E_{c o l}=3.47 \mathrm{eV}$ and Molecular Angular Momentum Randomly Selected from a Boltzmann Distribution at $40000 \mathrm{~K}$

\begin{tabular}{|c|c|c|c|}
\hline$v_{a 1}^{\prime}, v_{a 2}^{\prime}, v_{a 3}^{\prime}$ & $v_{b 1}^{\prime}, v_{b 2}^{\prime}, v_{b 3}^{\prime}$ & Prob. & Cross Section $\left(\AA^{2}\right)$ \\
\hline $\begin{array}{lll}1 & 1 & 0\end{array}$ & $\begin{array}{lll}1 & 1 & 0\end{array}$ & 0.05323 & 72.0 \\
\hline $\begin{array}{lll}1 & 2 & 0\end{array}$ & $\begin{array}{lll}1 & 1 & 0\end{array}$ & 0.04945 & 67.0 \\
\hline $\begin{array}{lll}1 & 1 & 0\end{array}$ & $\begin{array}{lll}0 & 1 & 0\end{array}$ & 0.04061 & 55.0 \\
\hline $\begin{array}{lll}2 & 1 & 0\end{array}$ & $\begin{array}{lll}1 & 1 & 0\end{array}$ & 0.02649 & 36.0 \\
\hline $\begin{array}{lll}2 & 0 & 0\end{array}$ & $\begin{array}{lll}1 & 1 & 0\end{array}$ & 0.02507 & 35.0 \\
\hline $\begin{array}{lll}1 & 3 & 0\end{array}$ & $\begin{array}{lll}1 & 1 & 0\end{array}$ & 0.02527 & 34.0 \\
\hline $\begin{array}{lll}1 & 1 & 0\end{array}$ & $\begin{array}{lll}0 & 2 & 0\end{array}$ & 0.02101 & 29.0 \\
\hline 120 & $\begin{array}{lll}0 & 1 & 0\end{array}$ & 0.01700 & 24.0 \\
\hline $\begin{array}{lll}2 & 0 & 0\end{array}$ & 120 & 0.01363 & 20.0 \\
\hline $\begin{array}{lll}2 & 1 & 0\end{array}$ & 120 & 0.01318 & 18.0 \\
\hline 140 & $\begin{array}{lll}1 & 1 & 0\end{array}$ & 0.01266 & 17.8 \\
\hline 130 & 120 & 0.01274 & 17.5 \\
\hline $\begin{array}{lll}1 & 1 & 0\end{array}$ & $\begin{array}{lll}0 & 3 & 0\end{array}$ & 0.01229 & 17.1 \\
\hline $\begin{array}{lll}1 & 2 & 0\end{array}$ & $\begin{array}{lll}1 & 2 & 0\end{array}$ & 0.01233 & 16.6 \\
\hline $\begin{array}{lll}1 & 2 & 0\end{array}$ & $\begin{array}{lll}0 & 2 & 0\end{array}$ & 0.01132 & 16.0 \\
\hline $\begin{array}{lll}2 & 0 & 0\end{array}$ & $\begin{array}{lll}0 & 1 & 0\end{array}$ & 0.01140 & 15.6 \\
\hline 130 & $\begin{array}{lll}0 & 1 & 0\end{array}$ & 0.00974 & 13.7 \\
\hline $\begin{array}{lll}2 & 1 & 0\end{array}$ & $\begin{array}{lll}0 & 1 & 0\end{array}$ & 0.00945 & 13.4 \\
\hline $\begin{array}{lll}0 & 2 & 0\end{array}$ & $\begin{array}{lll}0 & 1 & 0\end{array}$ & 0.00876 & 12.6 \\
\hline $\begin{array}{lll}3 & 0 & 0\end{array}$ & $\begin{array}{lll}1 & 1 & 0\end{array}$ & 0.00872 & 11.7 \\
\hline $\begin{array}{lll}0 & 1 & 0\end{array}$ & $\begin{array}{lll}0 & 1 & 0\end{array}$ & 0.00795 & 11.3 \\
\hline 220 & $\begin{array}{lll}1 & 1 & 0\end{array}$ & 0.00791 & 10.7 \\
\hline $\begin{array}{lll}1 & 1 & 0\end{array}$ & $\begin{array}{lll}0 & 4 & 0\end{array}$ & 0.00815 & 10.6 \\
\hline 150 & $\begin{array}{lll}1 & 1 & 0\end{array}$ & 0.00763 & 10.5 \\
\hline $\begin{array}{lll}2 & 1 & 0\end{array}$ & 130 & 0.00722 & 10.0 \\
\hline $\begin{array}{lll}2 & 1 & 0\end{array}$ & $\begin{array}{lll}2 & 0 & 0\end{array}$ & 0.00682 & 10.0 \\
\hline $\begin{array}{lll}2 & 1 & 0\end{array}$ & $\begin{array}{lll}0 & 2 & 0\end{array}$ & 0.00677 & 9.6 \\
\hline $\begin{array}{lll}1 & 4 & 0\end{array}$ & 120 & 0.00665 & 9.5 \\
\hline $\begin{array}{lll}2 & 0 & 0\end{array}$ & 130 & 0.00665 & 9.0 \\
\hline
\end{tabular}

$\mathrm{O}_{\text {gas }}+\mathrm{O}_{a d} *$ silica $\rightarrow \mathrm{O}_{2, g a s}(v, j)+$ silica

and

$\mathrm{H}_{\text {gas }}+\mathrm{H}_{a d} *$ silica $\rightarrow \mathrm{H}_{2, g a s}(v, j)+$ silica

In the dynamics simulations of both reactions, the preadsorbed atom (i.e., $\mathrm{O}_{a d}$ or $\mathrm{H}_{a d}$ ) is located over the central $\mathrm{Si}$ atom (the most important adsorption site), while the initial $(\mathrm{x}, \mathrm{y})$ coordinates of the impinging gas atom (i.e., $\mathrm{O}_{\text {gas }}$ or $\mathrm{H}_{\text {gas }}$ ) are chosen randomly over all available positions inside the surface unit cell. Incoming $\mathrm{O}_{\text {gas }}$ atoms impinge on the surface at $E_{\mathrm{col}}$ values falling in the range $(0.008-3.8) \mathrm{eV}$, while for the $\mathrm{H}_{\text {gas }}$ atoms this occurs in the range (0.04-3.0) $\mathrm{eV}$. Despite the fact that the surface temperature $\left(\mathrm{T}_{S}\right)$ in the case of $s c l$ calculations is $\mathrm{T}_{S}=1000 \mathrm{~K}$ and in the case of $c l$ calculations is $\mathrm{T}_{S}=1100 \mathrm{~K}$, the reported cross sections can still be directly compared because the dependence of the reactivity on $\mathrm{T}_{S}$ is small. An important parameter of the process of a gas-phase atom impinging on a solid substrate are the orientation angles $\left(\theta_{v}, \varphi_{v}\right)$, with $\theta_{v}$ being the angle between the incoming atom velocity vector and the normal to the surface plane and $\varphi_{v}$ being the azimuthal orientation angle over the surface plane. For our calculations, we have considered the normal incidence angle (i.e., $\theta_{v}=0^{\circ}$ ). At this incidence angle, $\varphi_{v}$ is undefined. From a theoretical point of view, for an E-R reaction, the reactive cross section $\left(\sigma_{E-R}\right)$ is usually defined as

$\sigma_{E-R}=\int_{0}^{2 \pi} \int_{0}^{b}{ }^{\max } P(b, \varphi) b d b d \varphi$

where $b$ is the impact parameter, $P(b, \varphi)$ is the opacity function and $\varphi$ is the orientation angle of the impinging particle. Such expression becomes simpler in the case of axial symmetry,

$\sigma_{E-R}=\int_{0}^{b_{\max }} P(b) b d b$

though, due to the usual cell symmetries of the solid surface, it is possible that $P$ depends on the angle. At the same time, in order to sample correctly the impact parameter $b(b=0$ for the initial position of $\mathrm{A}_{a d}$ on the cell), according to the standard Monte Carlo method its value should be taken from a $b^{2}$ uniform distribution between 0 and $b_{\max }^{2}$. Frequently, due to the fact that we are interested not only in the E-R process but also in the different competitive processes, a uniform cell sampling is carried out for the incoming atom. This allows to calculate all reaction probabilities $P_{i}$ (for the $i$ th channel) with a comparable quality. Therefore, for each process in the case of an orthogonal unit cell (i.e., as is the case of the $\beta$-cristobalite surface discussed in this paper),

$\sigma_{i}=\int_{0}^{a_{x}} \int_{0}^{a_{y}^{y}} P_{i}(x, y) d x d y \approx \frac{A}{N} \sum_{j=1}^{N} w_{i}\left(\chi_{1}, \chi_{2}\right)_{j}$ 
where $a_{x}$ and $a_{y}$ are the lattice parameters, $A$ is the unit cell area $(A=a \cdot b), N$ is the total number of selections made and $w_{i}\left(\chi_{1}, \chi_{2}\right)$ is either equal to 1 or 0 for all the $j$ initial selection (i.e., trajectory) depending on whether the $i$ channel is reached or not. The two random numbers $\chi_{1}$ and $\chi_{2}$ are selected in a way that $0 \leq \chi_{1}, \chi_{2} \leq 1$, with $x=\chi_{1} \cdot a_{x}$ and $y=\chi_{2} \cdot a_{y}$. Finally, the probability of each $P_{i}$ channel is taken equal to the $N_{i} / N$ fraction of trajectories assigned to the $i$ th-channel.

If the surface corresponds to a periodic structure, only a minimum unrepeated unit cell should be sampled. This is entirely correct for a clean surface while some corrections should be introduced for a pre-covered one. Thus, if only one atom is adsorbed into the unit cell, the previous expressions holds. However, for higher coverages, alternative targets (i.e., $a d$ atoms) are introduced. Therefore, if the same total unit cell is used, the calculated cross section (e.g., for the E$\mathrm{R}$ reaction) should be divided by the number of equivalent targets. Likewise an atomic adsorption process, the corresponding cross section should be divided by the number of equivalent adsorption sites.

In Fig. (7) the cross sections $\sigma_{E-R}$ calculated for the oxygen atom recombination (i.e., reaction (11)) using both scl (semiclassical [64, 65]) and $c l$ (classical [66]) dynamics methods are plotted together with the ones obtained for the hydrogen atom recombination (i.e., reaction (12)) using the $s c l$ technique. In the following, $\sigma_{E-R s c l}$ refers to the calculated cross section using the $s c l$ approach, while $\sigma_{E-R c l}$ refers to the cross section obtained using the $\mathrm{cl}$ approach. In the case of oxygen atom recombination (see Fig. 7), there is a good agreement between the $\mathrm{scl}$ and $\mathrm{cl}$ cross sections at low collision energies $(<0.1 \mathrm{eV})$. At intermediate energies $(\simeq 0.1-0.5 \mathrm{eV})$ the agreement seems to be much worse, improving again at higher energies $(\simeq 1$ $\mathrm{eV}$ ). However, additional $\mathrm{cl}$ and $s c l$ cross sections would be necessary to compare appropriately this behaviour (see, eqs. (11) and (12), respectively). Using only the scl method we can infer that the energy threshold for the two processes is different. In particular, the recombination reaction for $\mathrm{H}$ is more efficient than that for $\mathrm{O}$. This depends on the fact that the pre-adsorbed $\mathrm{H}$ atom is more weakly bound than the $\mathrm{O}$ atom and is therefore more prone to recombine. The two reactions exhibit also a different trend with collision energy. In fact, while in the case of oxygen recombination, $\sigma_{E-R s c l}$ decreases as $E_{\text {col }}$ increases, the opposite is true for hydrogen recombination whose $\sigma_{E-R s c l}$ increases with $E_{\text {col }}$ up to a maximum value of $5.0 \AA^{2}$ at collision energies around 0.4 $\mathrm{eV}$. Another important aspect of the recombination reactions, of relevance for the TPS problem, is the reaction exothermicity partitioning between the internal degrees of freedom of the new-formed $\mathrm{O}_{2}$ and $\mathrm{H}_{2}$ molecules and the surface. In Fig. (8) we compare the percentage of energy transferred to the internal degrees of freedom and to the

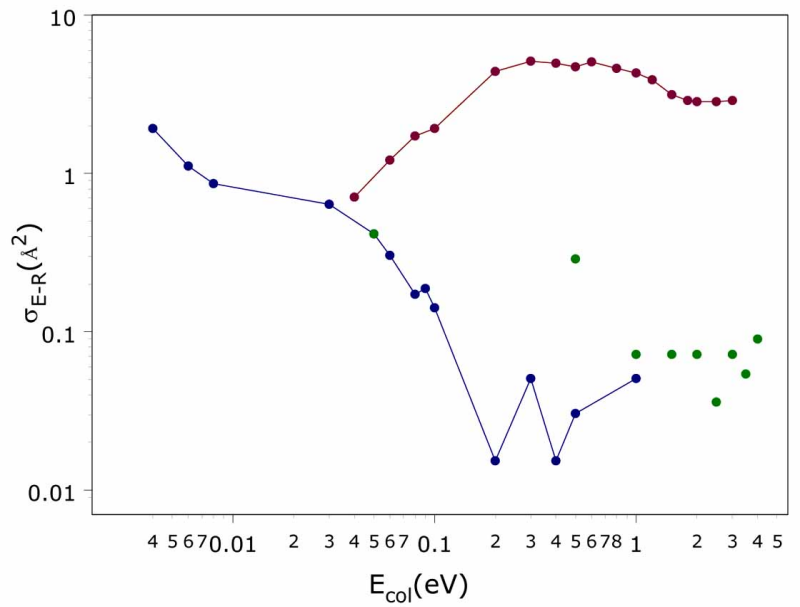

Fig. (7). $\sigma_{E-R}$ values calculated on the on $\beta$-cristobalite PES using semiclassical approaches for oxygen (blue dots and lines) and hydrogen (red dots and lines) atoms recombination. The $\sigma_{E-R}$ values calculated for the oxygen atom (green dots) recombination using the classical approach is also shown.
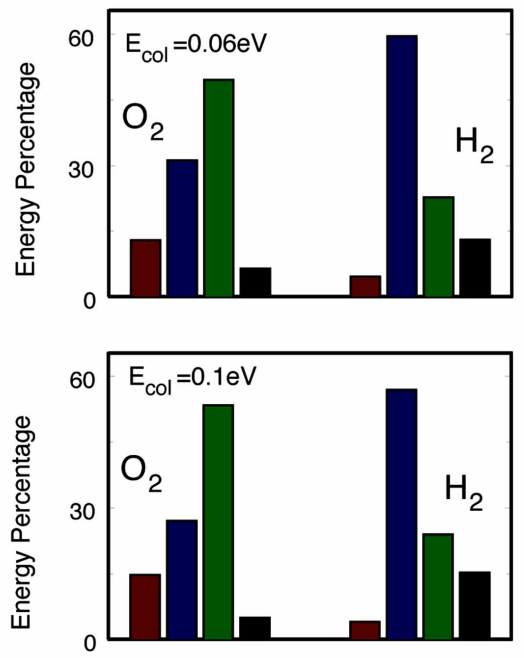

Fig. (8). Reaction exothermicity percentage transferred to vibrational (blue bar), rotational (green bar), translational (black bar) motion of the formed $\mathrm{O}_{2}$ and $\mathrm{H}_{2}$ molecules and to the phonons surface (red bar), using the semiclassical approach for $E_{\text {col }}=0.06$ and $0.1 \mathrm{eV}$ and $\mathrm{T}_{s}=1000 \mathrm{~K}$.

surface phonons using the $s c l$ approach. The comparison is made at two collision energies ( $E_{\mathrm{col}}=0.06$ and $0.1 \mathrm{eV}$ ). From Fig. (8) it can be inferred that, in the case of the formation of the $\mathrm{O}_{2}$ molecules, the largest fraction of available energy is disposed into rotation, whereas in the case of the formation of $\mathrm{H}_{2}$ it is disposed into vibration. Besides, the coupling with the surface silica atoms is more active in the case of oxygen recombination because of the different molecular masses of $\mathrm{O}$ and $\mathrm{H}$. In fact, in the formation of the $\mathrm{O}_{2}$ molecules, the percentage of energy exchanged with the surface phonons reaches a value of $15 \%$, about three times larger than the energy transferred to the surface in the case of $\mathrm{H}_{2}$ formation. The vibrational energy of the newly formed molecules is 
higher for $\mathrm{H}_{2}$ than for $\mathrm{O}_{2}$. This can also be inferred from the vibrational distributions, $P(v)$, reported in Fig. (9). The two distributions were obtained at low energy. In particular, the $\mathrm{O}_{2}$ distribution (see Fig. 9a) is obtained for a thermal condition $\left(T=T_{g a s}=T_{S}=1000 \mathrm{~K}\right.$ that could well correspond to an $E_{\mathrm{col}}$ of $0.12 \mathrm{eV}$ if $3 / 2 \mathrm{k}_{B} T$ is assumed for this kind of condition) and using the $c l$ method on the CRP PES [66]. On the contrary, the $\mathrm{H}_{2}$ distribution (Fig. 9) is obtained for $E_{\text {col }}=0.06 \mathrm{eV}$ using the $s c l$ approach on the recently reported PES of Ref. [67]. The main difference between the two distributions is that for the $\mathrm{O}_{2}$ molecules it is thermal while for the $\mathrm{H}_{2}$ ones it is inverted. Moreover, the number of vibrational states implied is larger in the case of $\mathrm{H}_{2}$ distribution $(0 \leq v \leq 12)$ than in the case of the $\mathrm{O}_{2}$ one $(0 \leq v \leq 8)$ although the collision energy of the incoming $\mathrm{H}$ atoms is lower than that of the $\mathrm{O}$ atoms. One has also to take into account that the vibrational levels present a higher separation in the case of $\mathrm{H}_{2}$ molecule due to a lighter mass. These distributions agree as well with the shape of the PESs. Thus, for the case of the $\mathrm{O}_{2}$ distribution the uppermost populated level $v=8$ corresponds to an internal energy of $\mathrm{E}(v=8, j=0) \approx 1.5 \mathrm{eV}$ (under harmonic oscillator and rigid rotor approximations) that agrees perfectly with the exothermicity of E-R process ((11)) on the CRP PES [66] ( $\Delta E_{E-R}=1.4 \mathrm{eV}$ ) the extra contribution from the initial $E_{\mathrm{col}}$ close to $0.12 \mathrm{eV}$. For the case of the $\mathrm{H}_{2}$ distribution the highest populated vibrational level $v=12$ corresponds to an internal energy of $\mathrm{E}(v=12, j=0) \approx 2.44 \mathrm{eV}$ that agrees also perfectly with the exothermicity of E-R reaction $((12))$ in the recently published PES [67] $\left(\Delta E_{E-R}=2.4 \mathrm{eV}\right)$ and the extra initial $E_{\mathrm{col}}=0.06 \mathrm{eV}$.
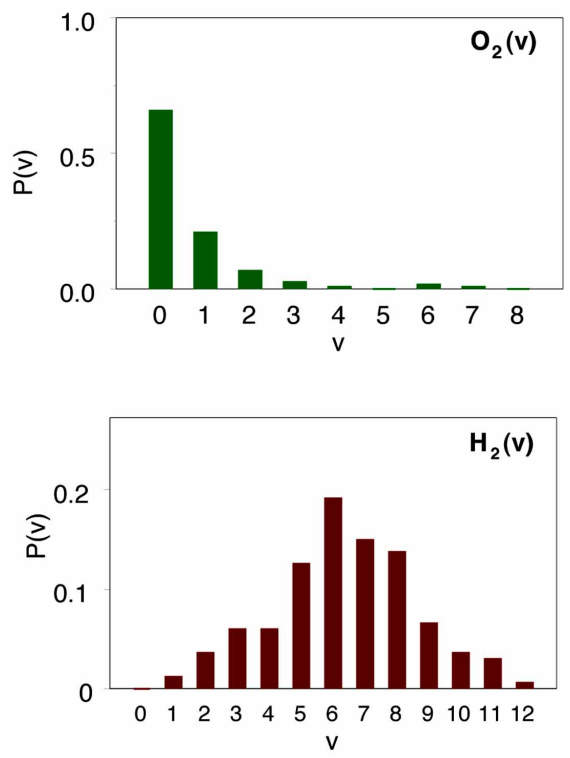

Fig. (9). Vibrational population distributions (normalized to unit) for $\mathrm{O}_{2}$ and $\mathrm{H}_{2}$ molecules formed by E-R reaction at $E_{\mathrm{col}}=0.13 \mathrm{eV}$ for the classical approach and $0.06 \mathrm{eV}$ for semiclassical approach, respectively.

\section{CONCLUSIONS}

In the present paper we report on the exploitation of the computing power made available by the European Grid infrastructure to carry out massive computations of the detailed cross sections of gas phase and gas surface collision processes for systems relevant to the modeling of planetary entries. The rigorous detailed quantum treatments has been recalled though for practical purposes (memory size and computing time) trajectory approaches have been preferred. For similar reasons semi-empirical formulations of the PESs have been adopted even if their parameters have been regularly used to tune their main features to that of rigorous electronic structure calculations. At the same time trajectory outcomes have been compared with those of other theoretical treatments and experimental data in order estimate their accuracy and support their validation.

The picture emerging from our work is that computational approaches to the microscopic modeling of the processes occurring during the atmospheric entry of spacecrafts are deemed to be successful if inserted into more global model treatments. The success of such approach is clearly ascribable to the use of Grid technologies which allow a detailed investigation of the efficiency of state-tostate processes through the use of the so called Grid Empowered Molecular Simulator without depending on large scale facilities.

\section{CONFLICT OF INTEREST}

The authors confirm that this article content has no conflicts of interest.

\section{ACKNOWLEDGEMENTS}

The authors acknowledge financial support from the Phys4entry FP7/2007-2013 project (Contract 242311), ARPA Umbria, INSTM, the EGI-Inspire project (Contract 261323), MIUR PRIN 2008 (2008KJX4SN 003), MIUR PRIN 2010-2011 (contract 2010ERFKXL_002), the ESAESTEC contract 21790/08/NL/HE and the Spanish CTQ2009-07647 and 2009SGR1041 projects. Computations have been supported by the use of Grid resources and services provided by the European Grid Infrastructure (EGI) and the Italian Grid Infrastructure (IGI) through the COMPCHEM Virtual Organization. Thanks are also due to the COST CMST European Cooperative Project CHEMGRID (Action D37). A. Lombardi thanks the Italian Consortium CINECA for the allocated computing time.

\section{REFERENCES}

[1] Sarma GSR. Relevance of aerothermochemistry for hypersonic technology. In: Capitelli M Ed. Molecular Physics and Hypersonic Flows NATO ASI Series C. Dordrecht: Kluwer Academic Publishers 1996; 482: pp. 1-20.

[2] Bertin JJ, Cummings RM. Critical hypersonic aerothermodynamics phenomena. Ann Rev Fluid Mech 2006; 38:129-57.

[3] Capitelli M. Non equilibrium vibrational kinetics. Berlin: Springer 1986.

[4] Capitelli M, Barsdley JN. Non equilibrium processes in partially ionized gases. New York: Plenum 1990.

[5] Kovalev VL, Kolesnikov AF. Experimental and theoretical simulations of heterogeneous catalysis in aerothermochemistry. Fluid Dyn 2005; 40: 669-93.

[6] Cacciatore M, Rutigliano M. Molecular dynamics simulation of surface processes: oxygen recombination on silica surfaces at high 
temperatures. In: Chazot O, Ed. Experiments, Modeling and Simulation of Gas-Surface Interactions of Reactive Flows in Hypersonic Flights. RTO-EN-AVT-142 2007; paper 5.

[7] Laganà A, Ochoa de Aspuru G, Garcia E. Quasi-classical and quantum rate coefficients for the $\mathrm{N}_{2}+\mathrm{N}_{2}$ reaction. Perugia: Stamperia Università di Perugia 1996.

[8] Verdicchio M, Pacifici L, Laganà A. Grid enabled high level $a b$ initio electronic structure calculations for the $\mathrm{N}_{2}+\mathrm{N}_{2}$ reaction. Lecture Notes Comp Sci 2012;7333: 371-86. Pacifici L, Verdicchio M, Faginas Lago N, Lombardi A, Costantini A. High Level Ab Initio study of the $\mathrm{N}_{2}+\mathrm{N}_{2}$ Reaction channel. J Comp Chem 2013; 34: 2668-76.

[9] Esposito F, Armenise I, Capitelli M. N-N $\mathrm{N}_{2}$ state to state vibrationalrelaxation and dissociation rates based on quasiclassical calculations. Chem Phys 2006; 331:1-8.

[10] Laganà A, Garcia E. Quasi-classical rate coefficients for the $\mathrm{H}+\mathrm{H}_{2}$ reaction. Perugia: Stamperia Università di Perugia 1996.

[11] Ceballos A, Garcia E, Rodriguez A, Laganà A. Quasi-classical rate coefficients for the $\mathrm{H}_{2}+\mathrm{H}_{2}$ reaction and dissociation. J Phys Chem Ref Data 2002; 31: 371-85.

[12] Esposito F, Gorse C, Capitelli M. Quasi-classical dynamics calculations and state-selected rate coefficients for $\mathrm{H}+\mathrm{H}_{2}(v, j) \rightarrow$ $3 \mathrm{H}$ processes: application to the global dissociation rate under thermal conditions. Chem Phys Lett 1999; 303: 636-40.

[13] Laganà A, Garcia E, Martinez T. Quasi-classical and quantum rate coefficients for the $\mathrm{O}+\mathrm{O}_{2}$ reaction. Perugia: Stamperia Università di Perugia 1996.

[14] Esposito F, Armenise I, Capitta G, Capitelli M. O-O ${ }_{2}$ state-to-state vibrational relaxation and dissociation rates based on quasiclassical calculations. Chem Phys 2008; 351: 91-8

[15] Available from : http://www.prace-ri.eu/ (last access 27jan2014)

[16] Laganà A, Riganelli $\mathrm{A}$, Gervasi O. On the Structuring of the Computational Chemistry Virtual Organization COMPCHEM. Lecture Notes Comp Sci 2006; 3980: 665-74.

[17] The European Grid Initiative (EGI). www.egi.eu (last access 27 Jan 2014).

[18] Gervasi O, Dittamo C, Laganà A. A Grid Molecular Simulator for E-Science. Lecture Notes Comp Sci 2005; 3470: 16-22.

[19] Laganà A, Gervasi O. A Priori Molecular Virtual Reality on EGEE Grid. Int J Quantum Chem 2009;110: 446-53. Laganà A, Costantini A, Gervasi O, Faginas Lago N, Manuali C, Rampino S. COMPCHEM: Progress Towards GEMS a Grid Empowered Molecular Simulator and Beyond. J Grid Comp 2010; 8: 571-86.

[20] Laganà A, Crocchianti S, Piermarini V. Towards a full dimensional exact quantum calculation of the $\mathrm{Li}+\mathrm{HF}$ reactive cross section. Lect Notes Comp Sci 2004; 3044: 422-31. Garcia E, Sánchez C, Saracibar A, Laganà A, Skouteris D. A detailed comparison of centrifugal sudden and $J$-shift estimates of the reactive properties of the $\mathrm{N}+\mathrm{N}_{2}$ reaction. Phys Chem Chem Phys 2009;11:11456-62. Rampino S, Skouteris D, Laganà A. Microscopic branching processes: The $\mathrm{O}+\mathrm{O}_{2}$ reaction and its relaxed potential representations. Int J Quantum Chem 2010; 110: 358.

[21] Manuali C, Costantini A, Laganà A, et al. Efficient Workload Distribution bridging HTC and HPC in Scientific Computing. Lect Notes Comp Sci 2012; 7333: 345-57.

[22] Freire E, Simon A, Lopez J, et al. Application Domain Accounting for EGI, 5th EGEE User Forum, Uppsala (SW) 2010. Available from : http://egee-uf5.eu-egee.org/

[23] Laganà A, Riganelli A. Reaction and Molecular Dynamics. Berlin: Springer Verlag 2000.

[24] Armenise I, Esposito F, Capitelli M. Dissociation-recombination models in hypersonic boundary layer flows. Chem Phys 2007; 336: 83-90. Armenise I, Esposito F. Dissociation-recombination models in hypersonic boundary layer $\mathrm{O}_{2} / \mathrm{O}$ flows. Chem Phys 2012; 398: 104-10. Capitelli M, Celiberto R, Esposito F, Laricchiuta A. Molecular Dynamics for State-to-State Kinetics of NonEquilibrium Molecular Plasmas: State of Art and Perspectives. Plasma Process Polym 2009; 6: 279-94.

[25] Minelli P, Esposito F, Bruno D, Capitelli M, Longo S. Extracting Cross Sections from Rate Coefficients: Application to Molecular Gas Dissociation. J Thermophys Heat Transfer 2011; 25: 374.

[26] Esposito F, Capitelli M. Selective vibrational pumping of molecular hydrogen via gas phase atomic recombination. J Phys Chem A 2009;113: 15307-14.
[27] Gamallo P, González M, Sayós R. Ab initio derived analytical fits of the two lowest triplet potential energy surfaces and theoretical rate constants for the $\mathrm{N}\left({ }^{4} S\right)+\mathrm{NO}\left(X^{2} \Pi\right)$ system. J Chem Phys 2003; 119: 2545 .

[28] Akpinar S, Armenise I, Defazio P, Esposito F, Gamallo P Petrongolo C, Sayós R. Quantum mechanical and quasiclassical Born-Oppenheimer dynamics of the reaction $\mathrm{N}_{2}\left(X^{1} \Sigma_{g}^{+}\right)+\mathrm{O}\left({ }^{3} P\right)$ $\rightarrow \mathrm{N}\left({ }^{4} S\right)+\mathrm{NO}\left(X^{2} \Pi\right)$ on the $\mathrm{N}_{2} \mathrm{O} \tilde{a}^{3} A^{\prime \prime}$ and $\tilde{b}^{3} A^{\prime} \tilde{\mathrm{O}}$ surfaces. Chem Phys 2012; 398: 81-89.

[29] Sayós R, Oliva C, González M. New analytical $\left({ }^{2} A^{\prime},{ }^{4} A^{\prime}\right)$ surfaces and theoretical rate constants for the $\mathrm{N}\left({ }^{4} S\right)+\mathrm{O}_{2}$ reaction. J Chem Phys 2002; 117: 670 .

[30] Esposito F. Dinamica Quasiclassical di Processi Collisionali Inelastici e Reattivi in Sistemi $\mathrm{H}+\mathrm{H}_{2}$ e $\mathrm{N}+\mathrm{N}_{2}$ rotovibrazionalmente risolti. $\mathrm{PhD}$ Thesis. Università degli Studi di Bari 1999.

[31] Esposito F, Capitelli M. Quasi-classical trajectory calculations of vibrationally specific dissociation cross sections and rate constants for the reaction $\mathrm{O}+\mathrm{O}_{2}(v) \rightarrow 3 \mathrm{O}$. Chem Phys Lett 2002; 364: 1807.

[32] Bose D, Candler GV. Thermal rate constants of the $\mathrm{N}_{2}+\mathrm{O} \rightarrow \mathrm{NO}$ $+\mathrm{N}$ reaction using ab initio ${ }^{3} A^{\prime \prime}$ and ${ }^{3} A^{\prime} \mathrm{O}$ potential energy surfaces. J Chem Phys 1996;104: 2825.

[33] Baulch DL, Bowman CT, Cobos CJ, et al. Evaluated Kinetic Data for Combustion Modeling: Supplement II. Phys Chem Ref Data 2005; 34: 757-1397.

[34] Shatalov OP. Recommended Data on Rate Constants of Physical and Chemical Processes in NĐO Atoms System. Inst. of Mechanics Avogadro Center. Russia: Moscow State University. Technical report 1987.

[35] Gamallo P, González M, Sayós R. Ab initio study of the two lowest triplet potential energy surfaces involved in the $\mathrm{N}\left({ }^{4} S\right)+\mathrm{NO}$

$\left(X^{2} \Pi\right)$ reaction. J Chem Phys 2003;118:10602.

[36] Baulch D, Cobos C, Cox R, et al. Evaluated kinetic data for combustion modeling. Supplement 1. J Phys Chem Ref Data 1994; 23: 847 .

[37] Gnoffo PA. Planetary entry gas dynamics. Ann Rev Fluid Mech 1999; 31: 459-94.

[38] Balakrishnan N, Vieira M, Babb J, Dalgarno A, Forrey R, Lepp S. Rate coefficients for ro-vibrational transitions in $\mathrm{H}_{2}$ due to collisions with He. Astrophys J 1999; 524: 1122.

[39] Muchnick P, Russek A. The $\mathrm{HeH}_{2}$ energy surface. J Chem Phys 1994; 100: 4336

[40] Boothroyd AI, Martin PG, Peterson MR. Accurate analytic $\mathrm{He}-\mathrm{H}_{2}$ potential energy surface from a greatly expanded set of $a b$ initio energies. J Chem Phys 2003; 119: 3187.

[41] Lee T-G, Rochow C, Martin R, Clarck TK, Forrey RC. Closecoupling calculations of low-energy inelastic and elastic processes in ${ }^{4} \mathrm{He}$ collisions with $\mathrm{H}_{2}$ : A comparative study of two potential energy surfaces. J Chem Phys 2005; 122: 024307.

[42] Orlikowski T. Close-coupling calculations of the cross sections and relaxation rates for ro-vibrational transitions in $\mathrm{H}_{2}$ colliding with He. Chem Phys 1981; 61: 405-13.

[43] Forrey RC, Balakrishnan N, Dalgarno A, Haggerty MR, Heller EJ. Quasi-resonant energy transfer in ultracold atom-diatom collisions. Phys Rev Lett 1999; 82: 2657-60.

[44] Paolini S, Ohlinger L, Forrey R. Hydrogen recombination due to collisions with He and Ar. Phys Rev A 2011; 83: 042713.

[45] Bartolomei M, Pirani F, Laganà A, Lombardi A. A full dimensional Grid empowered simulation of the $\mathrm{CO}_{2}+\mathrm{CO}_{2}$ processes. J Comp Chem 2012; 33: 1806-19.

[46] Cappelletti D, Pirani F, Bussery-Honvault B, Gomez L, Bartolomei M. A bond-bond description of the intermolecular interaction energy: the case of weakly bound $\mathrm{N}_{2}-\mathrm{H}_{2}$ and $\mathrm{N}_{2}-\mathrm{N}_{2}$ complexes. Phys Chem Chem Phys 2008; 10: 4281-93.

[47] Lombardi A, Faginas Lago N, Laganà A, Pirani F, Falcinelli S. A Bond-Bond Portable Approach to Intermolecular Interactions: Simulations for N-methylacetamide and Carbon Dioxide Dimers. Lecture Notes Comput Sci Part 1 2012; 7333: 387-400.

[48] Lombardi A, Laganà A, Pirani F, Lago FN, Palazzetti F. Carbon oxides in gas flows and earth and planetary atmospheres: State-to- 
state simulations of energy transfer and dissociation reactions. Available from : http://link.springer.com/chapter/10.1007

[49] Faginas Lago N, Albertí M, Laganà A, Lombardi A. Water $\left(\mathrm{H}_{2} \mathrm{O}\right)_{m}$ or Benzene $\left(\mathrm{C}_{6} \mathrm{H}_{6}\right)_{n}$ Aggregates to Solvate the $\mathrm{K}^{+}$? Lecture Notes Comput Sci Part 1 2013; 7971: 1-5.

[50] Falcinelli S, Rosi M, Candori P, Vecchiocattivi F, Bartocci A, Lombardi A, Faginas-Lago N, Pirani F. Modeling the intermolecular interactions and characterization of the dynamics of collisional autoionization processes. Lecture Notes Comput Sci Part 1 2013; 7971: 69-83.

[51] Albertí M, Faginas Lago N, Laganà A, Pirani F. A portable intermolecular potential for molecular dynamics studies of NMANMA and NMA- $\mathrm{H}_{2} \mathrm{O}$ aggregates. Phys Chem Chem Phys 2011;13: 8422-32.

[52] Faginas Lago N., Albertì M., Costantini A., Pacifici L. An innovative synergistic Grid approach to the computational study of protein aggregation mechanisms. J. Molecular Modeling 2014; accepted.

[53] Su T-M, Palazzetti F, Grossi G, Aquilanti V. Molecular alignment and chirality in gaseous streams and vortices. Rendiconti Lincei 2013; 24(3): 291-7.

[54] Palazzetti F, Tsai P-Y, Lombardi A, et al. Aligned molecules: chirality discrimination in photodissociation and in molecular dynamics. Rendiconti Lincei 2013; 24(3): 299-308.

[55] Carter, S, Murrell, JN. Analytical Two-valued Potential Energy Functions for the Ground State Surfaces of $\mathrm{CO}_{2}\left(\tilde{\mathrm{X}}^{1} \Sigma_{g}^{+}\right)$and $\mathrm{CS}_{2}$

$\left(\tilde{X}^{1} \Sigma_{g}^{+}\right)$. Chem Acta 1984; 57: 355-365.

[56] Faginas-Lago N, Lombardi A, Pacifici L, Costantini A. Design and Implementation of a Grid Application for Direct Calculations of Reactive Rates. Comput Theor Chem 2013; 1022: 103-7.

[57] Pacifici L, Verdicchio M, Faginas Lago N, Costantini A. A high level ab initio study of the $\mathrm{N}_{2}+\mathrm{N}_{2}$ reaction channel. J Comp Chem 2013; 34(31): 2668-76.

[58] Lombardi A, Faginas-Lago N, Costantini A, Pacifici L. Modeling of energy transfer from vibrationally excited $\mathrm{CO}_{2}$ molecules: cross sections and probabilities for kinetic modeling of atmospheres, flows, and plasmas. J Phys Chem A 2013;117(45): 11430-40

[59] Laub B, Venkatapathy E. Thermal protection system technology and facility needs for demanding future planetary missions. International workshop on planetary probe atmospheric entry and descent trajectory analysis and science. Lisbon: Portugal 2003.

[60] Cacciatore M, Rutigliano M, Billing GD. Eley-Rideal and Langmuir-Hinshelwood recombination coefficients for oxygen on silica surfaces. J Thermophys Heat Transfer 1999;13:195-203.
[61] Barbato M, Reggiani S, Bruno C, Muylaert J. Model for heterogeneous catalysis on metal surfaces with application to hypersonic flows. J Thermophys Heat Transfer 2000;14: 412-420.

[62] Morón V, Gamallo P, Sayós R. DFT and kinetics study of $\mathrm{O} / \mathrm{O}_{2}$ mixtures reacting over a graphite (0001) basal surface. Theor Chem Acc 2011;128: 683-94.

[63] Morón V, Gamallo P, Martin-Gondre L, Crespos C, Larregaray P, Sayós R. Recombination and chemical energy accommodation coefficients from chemical dynamics simulations: $\mathrm{O} / \mathrm{O}_{2}$ mixtures reacting over a $\beta$-cristobalite (001) surface. Phys Chem Chem Phys 2011;13:17494-504.

[64] Billing GD. Dynamics of Molecule-Surface Interactions. New York: John Wiley \& Sons 2000.

[65] Cacciatore M, Billing GD. Dynamical relaxation of $\mathrm{H}_{2}(v j)$ on a copper surface. Surf Sci 1990; 232: 35-50.

[66] Arasa C, Morón V, Busnengo HF, Sayós R. Eley-Rideal reaction dynamics between $O$ atoms on $\beta$-cristobalite (100) surface: A new interpolated potential energy surface and classical trajectory study. Surf Sci 2009; 603: 2742-51.

[67] Gamallo P, Rutigliano M, Orlandini S, Cacciatore M, Sayós R. Molecular dynamics study of hydrogen atom recombination over silica, based on a new analytical DFT potential energy surface. AIP Conf Proc 2012;1501:1129-36.

[68] Rutigliano M, Zazza C, Sanna N, Pieretti A, Mancini G, Barone V, Cacciatore M. Oxygen adsorption on $\beta$-cristobalite polymorph: $a b$ initio modeling and semiclassical time-dependent dynamics. J Phys Chem A 2009;113:15366-75.

[69] Arasa C, Gamallo P, Sayós R. Adsorption of atomic oxygen and nitrogen at $\beta$-cristobalite (100): a density functional theory study. J Phys Chem B 2005;109:14954-64.

[70] Rutigliano M, Zazza C, Orlandini S, Sanna N, Barone V, Cacciatore M. Oxygen Atoms and Molecules on Silica Surfaces: collisional data relevant to aerospace In: Catalytic Gas-Surface Interactions, NATO Applied Vehicle Technology Panel AVT-199, STO-MP-AV-T199 2012; pp. 5-1- 5-28.

[71] Sayós R, Morón V, Arasa C, Busnengo HF. Theoretical dynamics study of several atomic and molecular oxygen processes over a silica surface. Proceedings of the 6th European Symposium on Aerothermodynamics for Space Vehicles (ESA SP-659), Versailles, France 2009. 